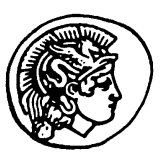

COMPUTERS \& EDUCATION

PERGAMON

\title{
"But my subject's different": a web-based approach to supporting disciplinary lifelong learning skills
}

\author{
Claire McAvinia* and Martin Oliver \\ Higher Education Research and Development Unit, Education and Professional Development, \\ University College London, 1-19 Torrington Place, London WC1E 6BT, UK
}

Accepted 16 November 2001

\begin{abstract}
Many new initiatives in Higher Education institutions choose to develop web sites to support their work, not least because web-based delivery of support materials from a central unit can help to deliver development materials via a single point of access, and 'on demand'. But this presents its own difficulties in terms of the selection and structure of generic material, and in making students aware of its existence. In this paper, the problem of designing a centrally managed web site (both in terms of structure and format) that adequately supports students across the institution will be discussed, and a strategy for developing a site that meets departmental needs will be presented, together with a discussion of the impact of this approach on the role of the developer. This is illustrated within the context of supporting Key Skills. 'Key' or 'transferable' skills are now recognised as being essential for most people in work and in life. Development of these skills is being encouraged at every level in education, and is demanded with increasing frequency by employers and professional bodies. Within Higher Education, the skills debate has prompted an examination of how students manage their own learning, and skills development initiatives encourage learners to seek ways of filling gaps in their knowledge and experience. However, at university level, it is frequently the case that explicit development of key skills must fall to students' spare time or to their extracurricular activities. This is partly explained by departmental traditions and experience, which may not encompass skills development. The UCL Key Skills site model, based on a 'core' website with customised departmental homepages, allows departments to make best use of central resources by 'personalising' the routes into these for their students. It also draws an important distinction in terms of the purpose of the web site, between management of information (for the central site) and pedagogy (for the departmental pages). The model helps to lay the foundations for graduates' lifelong learning by encouraging skills development, within a discipline-specific setting. The benefits of this approach - which included greatly increased levels of engagement and raised awareness amongst staff - are illustrated by a case study from the UCL Geography department, and wider applications of the model as a way of supporting centralised initiatives are discussed. (C) 2001 Published by Elsevier Science Ltd.
\end{abstract}

Keywords: Applications in subject areas; Lifelong learning; Teaching/learning strategies; Pedagogical issues

* Corresponding author. Tel.: +44-20-7679-1935; fax: +44-20-7679-1715.

E-mail address: c.mcavinia@ucl.ac.uk (C. McAvinia). 


\section{Introduction}

Web-based resources are often seen as an effective way of supporting new initiatives in Higher Education. Examples of this approach vary widely, from concerns such as writing across the curriculum in the USA (e.g. Reiss, Selfe, \& Young, 1998) to issues of access in Australia (e.g. McLoughlin \& Oliver, 2000) to transferable skills development in the UK (e.g. Drew, Shaw, \& Mowthorpe, 2000). However, this strategy remains problematic. In addition to the normal difficulties of web design, such as usability or the development of appropriate conceptual structures that can be used to manage information effectively, the introduction of new initiatives is likely to conflict with established cultures. As a result, if web-based support for central initiatives is to be effective, its design must be sensitive to the context in which it is to be used and the specific tensions attendant upon its adoption. This implies that the role of the designer needs to be different when working with such initiatives than when creating self-contained applications or resources within specific departmental cultures. In this case, it is argued, the role must be extended, from the technical development, conceptualisation and information management that it normally requires to include elements of social and contextual investigation.

This strategy has been tested in the context of supporting the implementation of Key Skills at UCL. In order to explain the context and tensions that informed the eventual site design, a summary will be given of issues driving the adoption of Key Skills in the UK, and surrounding the implementation of these initiatives in Higher Education. Based on this, examples of webbased support for Key Skills will be reviewed, and a model proposed that addresses the contextual and organisational shortcomings of previous approaches. This will be illustrated with a case study, showing the relationship between the central site (designed as a managed resource base) and specially developed 'tailored' front-ends created to fit in with specific departmental cultures. The wider relevance of this approach to other centrally driven initiatives will then be considered.

\section{Key Skills and lifelong learning}

The idea that there are certain 'core' or 'general' skills that people acquire through work and education, and which are distinct from specialist knowledge, is not a new one. Drew (1998) offers a useful history of the debate about skills in Britain, and suggests that since the 1960s and 1970s this has been articulated in terms of vocational or work-related skills, and whether or not it is the responsibility of state education to deliver them.

Current thinking at government level is that transferable or 'key' skills ought to be encouraged at every level in education - from schools to universities, and beyond in continuing professional development. Although there has been extensive debate about which skills are essential or 'key', one that occurs frequently is 'management of own learning'. Importantly, this skill links to the related areas of recording achievement, reflecting on progress and action planning, which are also being promoted at each level of education and by professional bodies in the workplace (Atkins, 1999; Stephenson, 1998).

These processes link to another important issue for education and economies in the twenty-first century: lifelong learning. Information and communication technologies, along with globalisation, 
have brought rapid change to the workplace (Candy, 2000). Lifelong learning and continuing professional development are arguably essential for graduates and other workers to remain competent. Therefore, the initiation of key skills development programmes and policies in Higher Education has in turn led to an examination of how students reflect on and record their development, and activities which are central to helping them become lifelong learners.

\section{Key Skills in Higher Education}

Although government and other national agencies take the view that key skills development should be encouraged, these initiatives have proved problematic in Higher Education. This is reflected in what Drew (1998) calls the 'Great Debate' about skills: many academics argue that it is not the role of Higher Education to deliver work-related skills, but the government may demand that universities justify their existence (and public funding) by doing exactly that.

The issue of what action universities are willing to take on skills is complicated. Firstly, employers are not consistent in their recruitment practices, and are not always able to predict which skills they are likely to need in the future (Drew, 1998). In addition, they still tend to recruit from older universities, in spite of the fact that post-1992 institutions are more likely to have included explicit employability skills elements in their degree programmes (Hesketh, 2000). Therefore, academic staff may justifiably feel wary of tailoring degree content and the skills students learn to include those specified by employers at any given time.

A further paradox arises from the overall position of the universities themselves: they are expected to widen participation, and have an increasingly varied student body which, now more than ever, demands good financial prospects on graduation and as such may demand employability skills within degree programmes. However, universities' funding and reputation rely largely on research status, which depends on academic specialisation and innovation, rather than the delivery of standardised employability skills in degree courses.

Moreover, the values of academic staff may not be the values of the skills initiative, and in many cases the development of transferable skills will be outside the traditions and experience of the department. In many cases, academic staff will perceive a skills programme or project as an attempt to introduce employability skills only, or to impose a set of politically motivated boundaries on what should and should not be covered in a given degree programme (Esland, 1996). In this context, many academic staff may not make the connection between skills development and the overall lifelong learning of the students concerned.

Skills initiatives themselves frequently face obstacles, at least in the early stages. One such obstacle is institutional debate over terminology, and the actual lists of key or essential skills that the institution wishes to focus on. This occurs partly because skills like communication and numeracy may already be embedded within subject disciplines, but in a subject-specific context. However, broadening the definition of the skill to apply to all students is difficult. For instance, being competent in the use of information technology may mean one thing to a Humanities student but something quite different to a Computer Sciences student (Hesketh, 2000).

Another aspect of skills development work which can make it hard to reconcile with traditional curricula is the need for skills development to be student-centred. It is difficult to fit this into what is, by necessity, a teacher-centred model, in much the same way that the introduction of student-centred 
negotiated learning contracts is often felt to be "practically impossible under the conditions prevailing at universities today" (Peters, 1998, p. 199). Additionally, the boundaries of the discipline and of the curriculum influence the perceived relevance and viability of specific skills development. There may be no apparent place in certain subject disciplines for particular skills: the example frequently given is that of numeracy skills in English, but there are many others. All this demonstrates the difficulties of covering areas that are not directly relevant to the student at that particular time within the context of coursework. This, together with the logistical problems of fitting extra modules or options into overcrowded curricula and resource issues in terms of staffing, materials and assessment of any kind, highlights the fact that integrating skills development into degree courses represents a considerable challenge.

However, in spite of these continuing debates, the majority of universities have instigated some kind of key skills development. Such approaches may well help universities to take a leading role in preparing graduates for the information society. Candy (2000) argues that since organisations are becoming more knowledge-based, academics as knowledge workers are ideally equipped to help students become lifelong learners in the information society. The skill of managing information in the wake of what he calls the 'information explosion', as well as the impact of developments like globalisation and unprecedented technological change, is now vital. He goes so far as to suggest that:

universities have a leadership role in producing graduates who are [...] attuned to the need for, and equipped with the skills of, continuing lifelong personal and professional development (Candy, 2000)

Some of the approaches proposed to achieve this, within the constraints outlined earlier, will now be explored further. This provides a necessary background for the development of an organisationally relevant and viable site design later in the paper.

\section{Implementing initiatives in skills for lifelong learning}

By encouraging the development of the skills of 'learning to learn' or 'improving own learning and performance', key skills initiatives help to accommodate the role of universities in helping students to become lifelong learners.

Whether key skills development contributes to graduate employability or not, it does provide students with a grounding in the processes necessary for lifelong learning. Key skills initiatives have prompted students to manage their own learning processes by:

- encouraging the articulation of existing skills and knowledge

- asking students to assess/judge their ability explicitly

- giving students a language and a format to describe and assess themselves

As a result of this initial articulation and expression of confidence and ability, the learner is able to identify personal learning objectives. The focus then shifts to development of these areas, followed by further reflection on changes in ability and the identification of the next areas for 
learning. This cycle sets in motion the habits that will enable a student to become a lifelong learner (cf. Kolb, 1984).

The models used by universities to implement and support these processes tend to move from the centre, or from a centrally located team, outwards, as illustrated by the following model (Drew et al., 2000):

1. Optional model: materials are recommended by tutors/course documents but the student is left to search them out and use them at their own discretion; no formal training is provided, and there is no formal relationship with specific parts of the course.

2. Directed model: the system is recommended by tutors/course documents, and students get formal intro/training in the use of materials, and are directed to them from time to time, but these are not strongly identified with specific parts of the course.

3. Integrated model: materials are recommended, formal introduction is provided, students are directed to materials at appropriate points of the course, and tutors associate materials within course/module delivery.

4. Contextualised model: materials are recommended and training provided; students are directed to use materials at appropriate points and tutors contextualise the system for use in their course.

In many cases, particular projects or initiatives have been introduced within the university to examine skills issues and to begin to deliver skills development to students. Skills delivery is therefore a formal, but extra-curricular activity - at least in the first instance when the initiative may be new to the institution. On a project or programme basis, and outside the curriculum, skills development is often the responsibility of a central team or unit such as a project team, the library, student support services, careers services and even the students' union.

\section{Using the web to support Key Skills}

The options for delivery of skills training or resources can also depend on where an institution places itself in the models outlined above: in some cases, the university may wish to see skills development embedded throughout the curriculum; others, for example, will want it to remain as a standalone, bolt-on option, or incorporated solely to support subject-specific study skills (Bennett, Dunne, \& Carré, 2000). Within institutions, particular departments may also adopt different levels of integration. Resources for delivery of skills are usually limited, both in terms of staffing and financial resources for the production and distribution of material.

In this context, web-based delivery of support materials from the central unit can overcome the twin problems of location and timing: the web provides a single point of access for all users independently of degree disciplines, and allows access 'on demand'. It is potentially a more costeffective use of resources, again in terms of key skills staff time and the costs of printing and distributing materials.

However, web-based delivery has disadvantages. Centralised sites may take the form of 'material put on the web': that is, staff with limited time may simply have had to make documentation available electronically, or copied text directly to a webpage without amending it or redesigning it 
for publication on a website. Text-heavy materials may predominate, along with lists of links to other similar websites. The problem addressed by the site here may be one of information management - which, without a structure that can also support students' learning, is unlikely to be effective in achieving its intended pedagogic aims.

On a practical level, the central site will require cross-campus publicity (which in turn demands staff time as well as financial outlay) to raise awareness of its existence. However, because it is a generic resource the students at which it is targeted may never seen its relevance to their particular courses.

By way of illustration, one initiative that aimed to provide a self-contained, generic, interactive web-based resource for key skills was Sheffield Hallam University's Key To Key Skills project (Drew et al., 2000). This was unusual in that it allowed for the development of web-based resources for key skills by a designated project team, members of which had already developed a set of well-regarded and popular paper-based materials for students. The project resulted in the production of a 'shell' that could be populated with the generic material bought under licence, or alternatively with an institution's own material. However, the usage of the web resource depended on the models of adoption outlined above: optional, directed, semi-integrated or fully integrated (ibid, p. 26). Although the final report noted that users perceived an enhancement to their learning processes and learning outcomes as well as their key skills from using the system, it also identified the need to take account of institutional cultures and the diverse needs of staff and students (ibid, p. 11).

A range of issues is apparent here: how can any centralised resource take account of the beliefs, values and methods of an institution, and in turn, of subject disciplines? If different disciplines not only have different cultures, but also different terminologies, how can centrally distributed materials be made relevant to particular groups of people? These problems remain unresolved in all the approaches outlined earlier. However, given the many problems associated with the integration of Key Skills in Higher Education, as discussed above, these issues cannot simply be ignored. Whilst tackling them cannot ensure the success of the associated initiative, not addressing them is likely to contribute to its failure.

The solution adopted for the Key Skills Pilot Project at UCL involves recognising these problems, working through them with specific groups of staff and attempting to design tailored solutions that are relevant, contextualised and meaningful to the department. This approach requires the designer to work within the context of departmental differences, rather than imposing a standard solution, as an essential component of its design (Fig. 1).

This model for web-based delivery of key skills at UCL is currently being implemented as part of a Key Skills Pilot Project (http://www.ucl.ac.uk/keyskills/), based in a central department (Education and Professional Development). It is based on a 'core' website, containing information and resources for three groups of users: staff, students and graduate students. In this model, resources may be generic skills materials, webpages, external weblinks, CAL materials, paperbased references or opportunities for skills development outside the curriculum (including student tutoring, mentoring, students' union activities and part-time work). In common with many key skills sites, the primary purpose of this core site is information management - in effect, it provides a 'portal' through which relevant materials can be accessed and opportunities to develop skills identified (Belcher, Place, \& Conole, 2000). The fully implemented site also includes a structured search mechanism with a number of fields to allow users to obtain a list of the available resources 
most relevant to their requirements at a particular time. The metadata search for key skills material allows the user to search by skill, discipline, type of resource and depth of resource. This prevents the main site from growing into an unwieldy list of links and references which would then require sorting. This in turn should enable students to make better use of the time they have available for skills development, although at the moment we do not have sufficient evaluation data to confirm this.

One innovative feature of this model is that it allows users to access this set of resources either through the generic, central key skills homepage, or via a tailored departmental key skills page. The generic central site provides a unified point of access, offering the economies of scale and efficiency of information management outlined earlier as an advantage of central web-based resources. However, in order to address the issues of disciplinary differences in terms of language, values, model of embedding, and so on, it proved necessary to provide multiple points of entry to this resource. These departmental homepages and are designed to:

- allow departments to prioritise certain skills and corresponding resources at relevant points in the course

- give students a particular route into resources

- provide a 'personalised' front-end to the site - a departmental, customised webpage rather than a central 'anonymous' one

- giving ownership of skills development to departments without adding to the workload of staff in those departments.

In terms of Drew et al.'s (2000) model of skills embedding, the development of these departmental pages represents a shift from optional to directed or even integrated provision. In doing so, it also adds an important new element to the web-based delivery of key skills, putting information at the disposal of the pedagogic aims of academic staff. Unlike the central site, which is structured by using a conceptual mapping of key skills to index resources, the design of these tailored sites requires knowledge of the curriculum and of the departmental context.

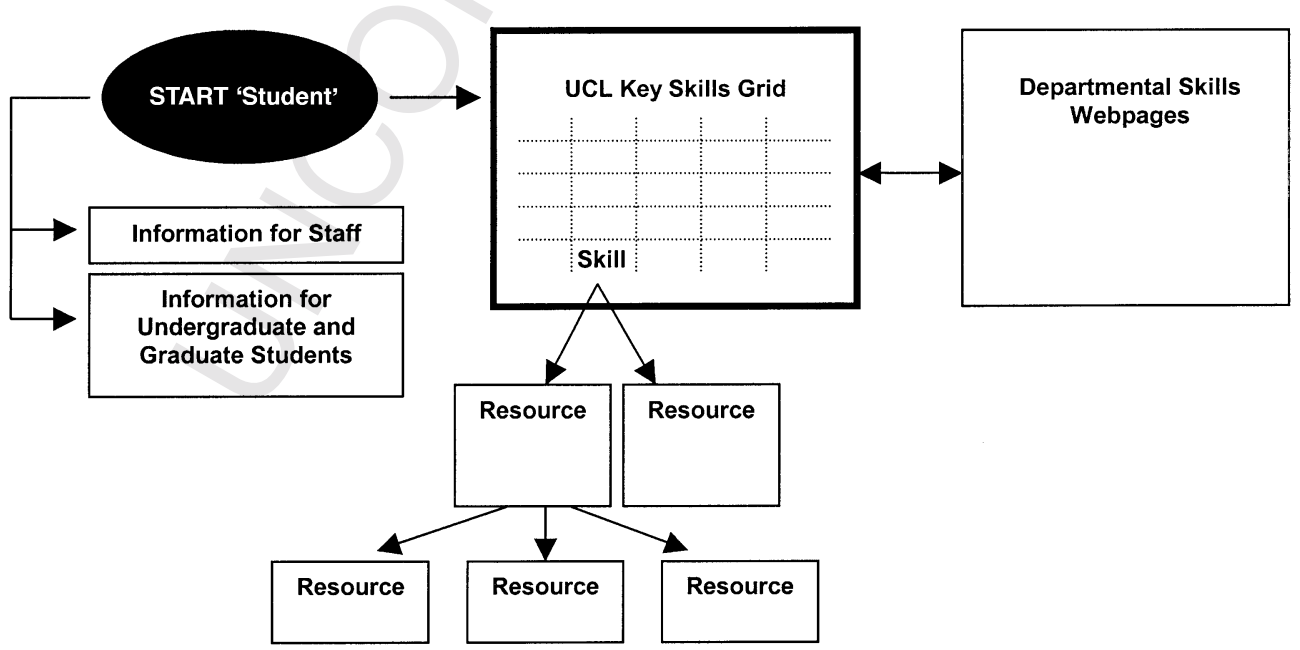

Fig. 1. The UCL Key Skills Model. 
Consequently, the development process incorporates a number of user-centred design practices, including preliminary interviews, building demonstration sites for approval and discussion, piloting and evaluation. Through these, the designer is required to develop their understanding of the skills and practices taught within the department, as well as the language used to describe these - information that usually remains tacit, and must be carefully elicited and interpreted given the contested meanings of terms in this area (Bennett et al., 2000). What this represents is an important shift in the role of the designer, extending it from a technical developer and librarian for the core site to include elements of ethnography.

\section{Key Skills for Geography}

A case study from UCL's Department of Geography illustrates the implementation of this model. The department was selected on the basis of its expressed interest in web-based resources to support skills development, which had arisen from its involvement in piloting a paper-based student profile that had been introduced as part of a wider UCL Key Skills Pilot (http://www. ucl.ac.uk/keyskills/geography/). This meant that members of staff, and most first year undergraduates, were already becoming familiar with the vocabulary and terminology of key skills, as well as with the process of expressing abilities and learning objectives for skills.

The department was approached to discuss the likely needs of its students and staff in terms of a set of 'customised' pages for key skills. The structure and format of these pages was discussed in a series of preliminary meetings between the designer and staff from the department. The department sought something that would fit in with their existing departmental web template, but which could be stored with the main key skills site in order to avoid complex local problems to do with web maintenance.

The resulting design was the homepage shown in Fig. 2-and it is clearly different to that of the main, central key skills site shown in Fig. 3.

In terms of the content of the pages, staff had suggested that numeracy skills be made the focus of the pilot webpages. One of their undergraduate courses, on data analysis and interpretation, called on a range of these skills and this had presented difficulties to a number of students. The range and level of materials to be provided on the skills webpages was discussed, and a variety of resources provided to cover basic number skills but also more complex numerical skills involved in data analysis. The pages also provided an opportunity to promote the use of existing networked packages within UCL, which represented an opportunity to encourage inter-disciplinary sharing of teaching and learning research that would otherwise have been unavailable to the department. The result was an organised, structured list of links to:

- networked interactive materials covering essential numerical skills (Key Skills Online: using calculators; decimals, fractions and percentages; formulae and algebra; handling data; statistics; measurements)

- CAL software for Geographers freely available on the web

- UCL's own Geomaths site (to help raise awareness of this existing resource)

- resources to help with using IT for data handling and analysis

- paper-based references for statistics and data analysis, including some generic and some discipline-specific references 


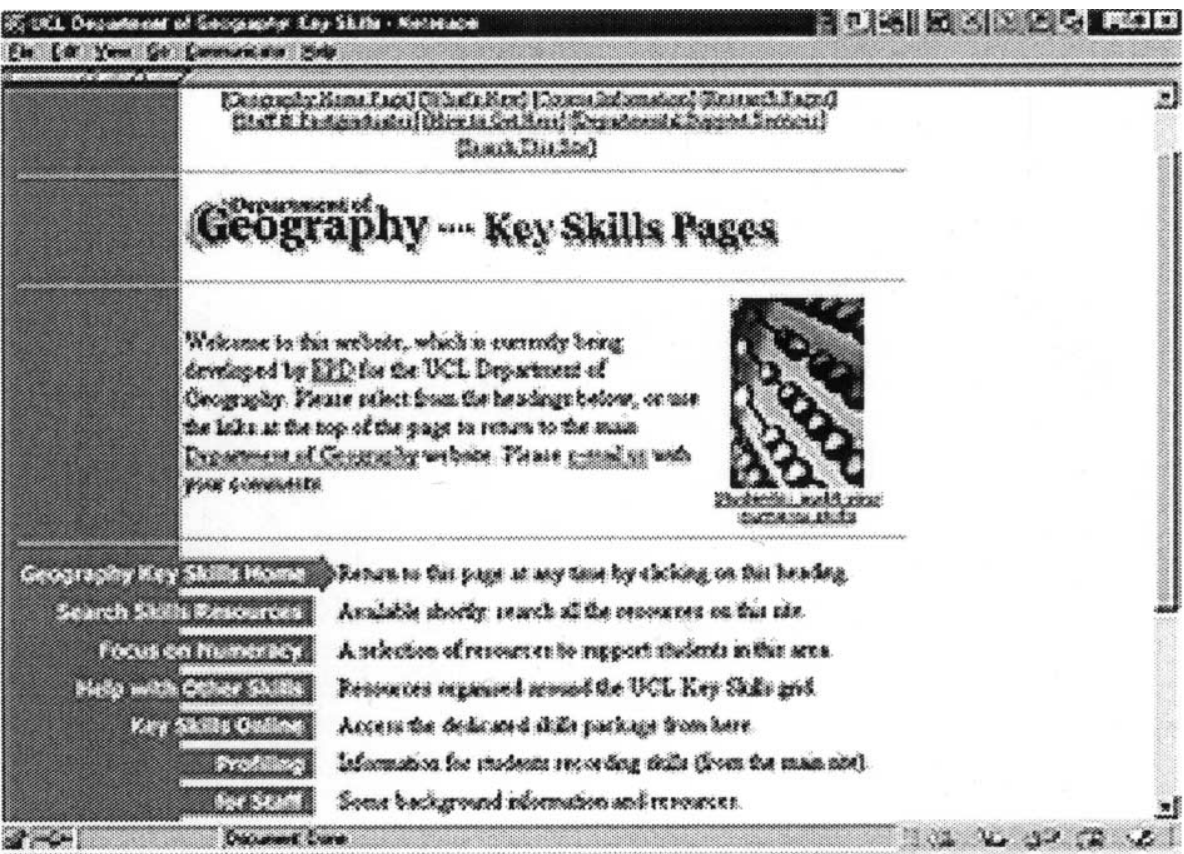

Fig. 2. The Geography Key Skills homepage.

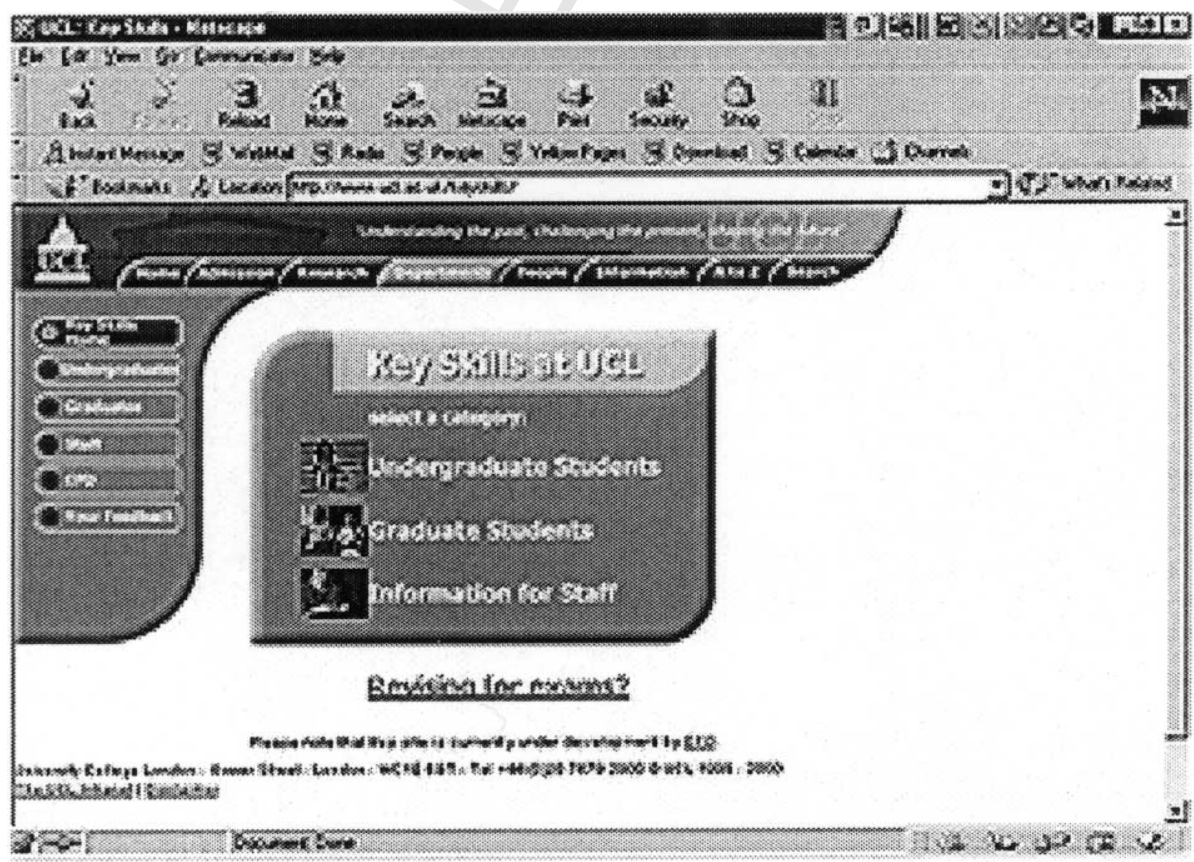

Fig. 3. The UCL Key Skills homepage. 
- online datasets which could provide practice in data-handling for geographers

- resources for other key skills related to the data analysis course itself: working in groups, report writing and general study skills.

Some of the resources were provided by the department in paper-based format: staff had made notes of useful websites, as well as paper-based references for disciplinary skills (e.g. Clark \& Wareham, 2000), and also asked for links to be made to their own webpages. Other generic and subject-specific resources were located by the web developer. The implementation of the skills pages provided an opportunity for this material to be organised and selected, and then linked to particular skills: this was something that staff in the department had not previously been able to do, partly because of time constraints, but also because the idea of using and developing students' key skills was relatively new to them. Importantly, it also raised the developer's awareness of useful materials previously unknown to them, allowing these to be classified, added to the central database and re-used in future customised pages.

\section{An evaluation of the site}

In order to evaluate the site, permission was sought to gain feedback from students involved in using the materials as part of their course. A suitable opportunity for this was identified in the form of a self-contained session involving around 25 students. The department ran a student-led session within the data analysis course, and e-mailed the students concerned with the link to the Geography Key Skills pages in advance of the session.

The student leader of this session used the site to prepare and commented that it had been very useful; this was in part a result of the way that it provided a self-contained study plan that structured their work on these topics and allowed them to discriminate further between the shortlist of resources. There was a positive response to the customised webpages from both staff and students, although eliciting detailed formal feedback from them has proved difficult due to the centralised nature of the web development project. However, a questionnaire on first-year students' use of the site was circulated to the 155 students in the year. This indicated that around half of the students had used the two websites (the generic and subject-specific) - well above the average number of students per department (c. 4-5 students from each department without its own tailored pages). This indicates a far greater awareness of the resource and more effective level of engagement when it is located within their subject discipline. In addition, over $95 \%$ of respondents found the self-assessment process to be helpful. However, responses to open questions did identify some problems with the approach, including the need for the profiling exercise to be integrated within personal tutoring sessions (i.e. a move from directed or integrated approach to a contextualised one) and the difficulty in determining the standards expected for these skills at university level.

Tutors were also invited to provide feedback on the use of the site; this was provided in the form of an agreed document of anonymised comments drawn up within the department and passed to the developers. These were broadly positive, and tutors have suggested that a similar approach be taken for analytical skills during the next academic year. In light of students' feedback, for example, it was proposed that personal tutorials be revised so as to properly contextualise 
the use of skills profiling. However, like the students, staff were concerned about providing feedback on standards.

Unless students get a sense of 'how their peers are doing' and what is expected of them at undergraduate level - through exchange of work, discussion about performance in a group or individually — these aspects of skills auditing are quite difficult for students to determine.

Importantly, some of the staff comments showed misgivings about the extent to which the web can be used to deliver skills development. This is considered to be a positive outcome-these tutors have moved from an initial position of thinking that web-based delivery is unproblematic, to an awareness of the issues involved, a consideration of alternative methods for supporting skills development, and a re-thinking of ways to integrating it further within the curriculum. This is supported by the production by Geography staff of a detailed map of skills in their degree courses, making key skills explicit in their curriculum - a positive indication of their intention to pursue skills development work with their students in a systematic way, and a reflection of a change in their departmental culture and discourse arising from the pilot.

\section{Conclusions}

Although web-based resources appear to be an efficient way of supporting centrally led initiatives such as key skills development, the reality is less straightforward. Because such initiatives arise from outside the disciplinary context of departments, the process of embedding them into the curriculum is complex and problematic, involving staff resistance, conceptual confusion and contested terminology. Consequently, designing support materials for such initiatives raises a set of distinctive challenges.

In this paper, the problems around embedding Key Skills have been described, and a strategy for design that addresses the above issues presented. This approach extends the role of the designer from the developmental and librarianship roles traditionally associated with portal sites to include elements of sociological investigation. This is achieved by meeting with (and ideally working alongside) members of departments to develop a sound understanding of their needs, concerns and pedagogy, and allows access routes to be developed that provide a sense of relevance, credibility and ownership of the generic, central resource.

This collaborative development also allows an important shift to be achieved in the design process - away from the effective management of information (supporting only an 'optional' model of embedding) and towards a more integrated provision of material. By structuring these tailored pages to support specific curricula, pedagogic goals can be met that would otherwise be beyond the scope of the site. However, it must be recognised that these benefits come at a price in terms of increased time required by the developer. Whether this is justified by the benefits achieved by the departmental sites, or is balanced out by time saved developing a series of separate developments, is a question that will need to be addressed on a case-by-case basis.

This approach - both in terms of the revised role of the designer, and the model of site implementation - has been found to be effective as a way of embedding key skills materials in a disciplinary context. Importantly, as illustrated earlier, the effects of this in terms of student 
engagement and the development of an informed critical perspective within the departmental can be considerable, reflecting the value of spending time to develop a culturally embedded, pedagogically relevant and linguistically appropriate resource. Whilst the general impact of this approach will require further investigation, it is clear that it represents a useful strategy for systematically supporting centrally led initiatives.

\section{Uncited references}

Bennett, Dunne, and Carré (1999), Brown (1997)

\section{References}

Atkins, M. (1999). Oven-ready and self-basting: taking stock of employability skills. Teaching in Higher Education, 4(2), 267-280.

Belcher, M., Place, E., \& Conole, G. (2000). Quality assurance in subject gateways: creating high quality portals on the Internet. Quality Assurance in Education, 8(1), 38-47.

Bennett, N., Dunne, E., \& Carré, C. (1999). Patterns of core and generic skill provision in higher education. Higher Education, 37, 71-93.

Bennett, N., Dunne, E., \& Carré, C. (2000). Skills development in Higher Education and employment. Buckinghamshire: OU Press.

Brown, A. (1997). The development of Key Skills across contexts and over time. Capability, 3(2), 16-20.

Candy, P. (2000). Knowledge Navigators and Lifelong Learners: producing graduates for the information society. Higher Education Research \& Development, 19(3), 261-277.

Clark, G., \& Wareham, T. (2000). Geography@University. Cheltenham: Crown Copyright/DfEE.

Drew, S. (1998). Key skills in Higher Education: background and rationale. SEDA Special no. 6. Birmingham: SEDA Publications.

Drew, S., Shaw, M. \& Mowthorpe, D. (2000) Key to Key Skills project final report. Available: http://www.shu.ac.uk/ keytokey/finalrep4.pdf.

Esland, G. (1996). Education, training and nation-state capitalism: Britain's failing strategy. In J. Avis, M. Bloomer, G. Esland, D. Gleeson, \& P. Hodkinson (Eds.), Knowledge and nationhood (pp. 40-70). London: Redwood Books.

Hesketh, A. (2000). Recruiting an Elite? Employers' perceptions of graduate education and training. Journal of Education and Work, 13(3), 245-271.

McLoughlin, C., \& Oliver, R. (2000). Designing learning environments for cultural inclusivity: a case study of indigenous online learning at tertiary level. Australian Journal of Educational Technology, 16(1), 58-72.

Peters, O. (1998). Learning and teaching in distance education: analyses and interpretations from an international perspective. London: Kogan Page.

Reiss, D., Selfe, D., \& Young, A. (1998). Electronic communication across the curriculum. USA: National Council of Teachers of English.

Stephenson, J. (1998). The concept of capability and its importance in Higher Education. In J. Stephenson, \& M. Yorke (Eds.), Capability and quality in Higher Education (pp. 1-14). London: Kogan Page. 